\title{
On Notivarg Propagator
}

\author{
M. Bakalarska, W. Tybor* \\ Department of Theoretical Physics \\ University of Eódź \\ ul.Pomorska 149/153, 90-236 Łódź, Poland
}

\begin{abstract}
The covariant propagator of the notivarg is found. It has the Feynmann - like form.
\end{abstract}

*Supported by Łódź University Grant No. 505/581 
1. The essential difficulty, we are faced with on calculating the gauge field propagator, is that the Lagrangian operator of the gauge theory is singular.The origin of the singularity is the gauge freedom of the theory. To remove it we impose the suitable gauge conditions. That allow us to obtain an effective action leading to the regular Lagrangian operator. The propagator is defined as the inverse operator to the Lagrangian one. The method works very well, for example, in the electrodynamics. It has been verified in the theory of the notoph $[1,2]$. However, the method is rather troublesome if the gauge field is a many component tensor. Such a situation is in the case of the notivarg that is described by the field with symmetry properties of a Riemann tensor.

We can follow another way to obtain the form of propagators. We consider the interaction of the gauge field with an external current that obeys some conservation law ensuring the gauge invariance for the interacting term. In the fixed Lorentz frame we perform the canonical analysis of the theory. Solving constraints we obtain the physical Hamiltonian. Its free part describes only the physical degrees of freedom. The interaction part is a sum of two terms. The first one describes the interaction of the physical components of the gauge field with the current components that are not restricted by the current conservation law. The second one describes the instant interactions, bilinear in the currents (for example, the Coulomb term $\rho \frac{1}{\Delta} \rho$ in the electrodynamics). Using the standard methods of the $\mathrm{S}$ - matrix formalism, with the help of this Hamiltonian, we can calculate the amplitude of the current - current interaction, squard in the coupling constant. On the other hand, the amplitude can be calculated using the covariant propagator. Taking into account the Lorentz properties of the field and the current and the law of the current conservation, we can predict the general form of the covariant propagator. The result of calculations must be independent of the calculation method. So, we can verify correctness of our prediction of the form of the covariant propagator.

2. The notivarg field $K^{\mu \nu \alpha \beta}$ interacting with the external Weyl current $j^{\mu \nu \alpha \beta}$ is described by the Lagrangian density [3]

$$
\mathcal{L}=-\left(\partial_{\sigma} K^{\sigma \nu \alpha \beta}\right)^{2}+\left(\partial_{\sigma} K_{\nu}^{\sigma \nu \alpha}\right)^{2}+\frac{1}{4} j^{\mu \nu \alpha \beta} K_{\mu \nu \alpha \beta},
$$

where $K^{\mu \nu \alpha \beta}$ has the symmetry properties of a Riemann tensor $K^{\mu \nu \alpha \beta}=$ $K^{\alpha \beta \mu \nu}=-K^{\nu \mu \alpha \beta}, \varepsilon_{\mu \nu \alpha \beta} K^{\mu \nu \alpha \beta}=0$. The Weyl current $j^{\mu \nu \alpha \beta}$ is the Riemann 
tensor obeying

$$
j_{\nu}^{\mu \nu \alpha}=0 .
$$

The conservation law for the Weyl current is

$$
\partial_{\mu} \partial_{\alpha} j^{\mu \nu \alpha \beta}=0
$$

The action determined by the Lagrangian (1) is invariant under the gauge transformations

$$
\begin{aligned}
\delta K^{\mu \nu \alpha \beta}= & \varepsilon^{\mu \nu \sigma \lambda} \varepsilon^{\alpha \beta \varphi \kappa} \partial_{\sigma} \partial_{\varphi} \omega_{\lambda \kappa}+g^{\mu \alpha}\left(\partial^{\nu} \eta^{\beta}+\partial^{\beta} \eta^{\nu}\right)+ \\
& +g^{\nu \beta}\left(\partial^{\mu} \eta^{\alpha}+\partial^{\alpha} \eta^{\mu}\right)-g^{\mu \beta}\left(\partial^{\nu} \eta^{\alpha}+\partial^{\alpha} \eta^{\nu}\right)-g^{\nu \alpha}\left(\partial^{\mu} \eta^{\beta}+\partial^{\beta} \eta^{\mu}\right)+ \\
& -2\left(g^{\mu \alpha} g^{\nu \beta}-g^{\mu \beta} g^{\nu \alpha}\right) \partial_{\sigma} \eta^{\sigma}
\end{aligned}
$$

where $\omega_{\alpha \beta}=\omega_{\beta \alpha}$ and $\eta_{\alpha}$ are gauge tensors.

In the covariant gauge

$$
K_{\nu}^{\mu \nu \alpha}=0, \quad \partial_{\mu} \partial_{\alpha} K^{\mu \nu \alpha \beta}=0
$$

the field equation has the form 1

$$
\square K^{\mu \nu \alpha \beta}=-\frac{1}{2} j^{\mu \nu \alpha \beta} .
$$

3. Let us consider the exchange of the notivarg between two external currents. The general structure of the amplitude describing the process in the second order of the perturbation theory is

$$
\begin{aligned}
A= & (-i)^{2}\left(\frac{a}{k^{2}} j^{\mu \nu \alpha \beta}(-k) j_{\mu \nu \alpha \beta}(k)+\frac{b}{k^{4}} k_{\mu} j^{\mu \nu \alpha \beta}(-k) k^{\sigma} j_{\sigma \nu \alpha \beta}(k)+\right. \\
& \left.+\frac{c}{k^{6}} k_{\mu} k_{\alpha} j^{\mu \nu \alpha \beta}(-k) k^{\sigma} k^{\kappa} j_{\sigma \nu \kappa \beta}(k)\right)
\end{aligned}
$$

where $a, b, c$ are number factors. The last term vanishes due to the conservation law (3). The second one has, in fact, the structure of the first one due to the following identity for the Weyl tensor

$$
j^{\mu \nu \alpha \beta} j_{\sigma \nu \alpha \beta}=\frac{1}{4} \delta_{\sigma}^{\mu} j^{\kappa \nu \alpha \beta} j_{\kappa \nu \alpha \beta} .
$$

\footnotetext{
${ }^{1}$ In Ref. [3] the right hand side of Eq. (7) reads $-\frac{1}{2} j^{\mu \nu \alpha \beta}$.
} 
Assuming the following form of the notivarg propagator

$$
\begin{aligned}
D_{\mu \nu \alpha \beta, \sigma \lambda \gamma \delta}(k)= & -\frac{1}{k^{2}} \frac{1}{8}\left(g_{\mu \sigma} g_{\nu \lambda} g_{\alpha \gamma} g_{\beta \delta}+g_{\mu \lambda} g_{\nu \sigma} g_{\alpha \delta} g_{\beta \gamma}+\right. \\
& +g_{\mu \gamma} g_{\nu \delta} g_{\alpha \sigma} g_{\beta \lambda}+g_{\mu \delta} g_{\nu \gamma} g_{\alpha \lambda} g_{\beta \sigma}-g_{\mu \lambda} g_{\nu \sigma} g_{\alpha \gamma} g_{\beta \delta}+ \\
& \left.-g_{\mu \sigma} g_{\nu \lambda} g_{\alpha \delta} g_{\beta \gamma}-g_{\mu \gamma} g_{\nu \delta} g_{\alpha \lambda} g_{\beta \sigma}-g_{\mu \delta} g_{\nu \gamma} g_{\alpha \sigma} g_{\beta \lambda}\right)
\end{aligned}
$$

we obtain the amplitude

$$
A=(-i)^{2}\left(-\frac{1}{8}\right) j^{\mu \nu \alpha \beta}(-k) D_{\mu \nu \alpha \beta, \sigma \lambda \gamma \delta}(k) j^{\sigma \lambda \gamma \delta}(k) .
$$

The number factor $-\frac{1}{8}$ follows from Eqs. (1) and (6)

$$
\frac{1}{4} j^{\mu \nu \alpha \beta} K_{\mu \nu \alpha \beta} \rightarrow \frac{1}{4} j^{\mu \nu \alpha \beta} D_{\mu \nu \alpha \beta, \sigma \lambda \gamma \delta}\left(-\frac{1}{2} j^{\sigma \lambda \gamma \delta}\right) .
$$

Using

(i) the current conservation law (3),

(ii) the decomposition of the Weyl current

$$
j^{\mu \nu \alpha \beta}=\left(\tau^{i j}, \sigma^{i j}\right), \quad i, j=1,2,3 ;
$$

where $\tau^{i j}$ and $\sigma^{i j}$ are symmetric and traceless tensors defined by

$$
\begin{aligned}
& j^{0 i 0 j}=\tau^{i j}, \\
& j^{0 i j k}=\varepsilon^{j k p} \sigma_{p}^{i}, \\
& j^{i j k l}=-\left(g^{i k} \tau^{j l}+g^{j l} \tau^{i k}-g^{i l} \tau^{j k}-g^{j k} \tau^{i l}\right) .
\end{aligned}
$$

(iii) the helicity decomposition of the symmetric traceless tensor $a^{i j}$

$$
a^{i j}=a^{i j}(0)+a^{i j}( \pm 1)+a^{i j}( \pm 2)
$$

where

$$
\begin{aligned}
a^{i j}( \pm 1) & =-\frac{1}{\Delta}\left(\partial^{i} a_{T}^{j}+\partial^{j} a_{T}^{i}\right), \\
a^{i j}(0) & =\frac{3}{2}\left(\frac{1}{\Delta} \partial^{i} \partial^{j}+\frac{1}{3} g^{i j}\right) a_{L}, \\
a^{i} & \equiv \partial_{j} a^{j i} \\
a_{L} & \equiv \frac{1}{\Delta} \partial_{i} a^{i},
\end{aligned}
$$


we obtain

$$
\begin{aligned}
A= & \frac{3}{2} \frac{\sigma_{L}(-k) \sigma_{L}(k)}{k^{2}}+2|\vec{k}|^{-4} \tau_{T i}(-k) \tau_{T}^{i}(k)+ \\
& +\frac{1}{4} k^{2} k_{0}^{-2}|\vec{k}|^{-2} \tau_{i j}( \pm 2,-k) \tau^{i j}( \pm 2, k) .
\end{aligned}
$$

From the canonical analysis [4] we obtain the physical Hamiltonian density describing interaction

$$
\begin{aligned}
\mathcal{H}_{i n t}= & \sqrt{\frac{3}{2}} \varphi \sigma_{L}+\frac{1}{2} \sigma_{m}^{n}( \pm 2) \frac{1}{\Delta} \sigma_{n}^{m}( \pm 2)+ \\
& +\frac{1}{8}\left(\frac{1}{\Delta} \partial^{0} \tau^{i j}( \pm 2)\right)\left(\frac{1}{\Delta} \partial^{0} \tau_{i j}( \pm 2)\right)-\frac{3}{8} \tau^{i j}( \pm 2) \frac{1}{\Delta} \tau_{i j}( \pm 2)+ \\
& -\left(\frac{1}{\Delta} \tau_{T}^{i}\right)\left(\frac{1}{\Delta} \tau_{T i}\right) .
\end{aligned}
$$

Two remarks are necessary:

1. The term (Eq. (34) in Ref. [4])

$$
-\frac{1}{4} \sigma_{m}^{n}( \pm 2) \frac{1}{\Delta} \sigma_{n}^{m}( \pm 2)
$$

is wrong. The right form is

$$
-\frac{1}{2} \sigma_{m}^{n}( \pm 2) \frac{1}{\Delta} \sigma_{n}^{m}( \pm 2)
$$

2. The new field $\varphi=\sqrt{6} S_{L}$ is introduced because the free physical Lagrangian has then the standard form

$$
\mathcal{L}_{\text {free }}=\frac{1}{2}\left(\partial_{\mu} \varphi\right)^{2}
$$

We recall that $S_{L}$ is a scalar component of the symmetric traceless tensor

$$
S^{i m}=-\frac{1}{4}\left(\varepsilon^{m}{ }_{j k} K^{0 i j k}+\varepsilon^{i}{ }_{j k} K^{0 m j k}\right) .
$$

The Hamiltonian density (10) in the momentum space is

$$
\begin{aligned}
\mathcal{H}_{\text {int }}= & \sqrt{\frac{3}{2}} \sigma_{L}(-k) \varphi(k)+\frac{1}{2}\left[2|\vec{k}|^{-4} \tau_{T i}(-k) \tau_{T}^{i}(k)+\right. \\
& \left.+\frac{1}{4} k^{2} k_{0}^{-2}|\vec{k}|^{-2} \tau_{i j}( \pm 2,-k) \tau^{i j}( \pm 2, k)\right]
\end{aligned}
$$


The current conservation law (3) is included in Eq. (11).

With the help of this Hamiltonian, using standard methods of the S - matrix formalism [5], we can obtain the amplitude of the current - current interaction via one notivarg exchange. We get exactly the amplitude (91). So, the Feynmann - like form (7) of the notivarg propagator is confirmed.

We are grateful to Prof. J. Rembieliński for interesting discussion. 


\section{References}

[1] L.C. Papaloucas, J. Rembieliński, W. Tybor, Acta Phys. Pol. B22, 429 (1991) .

[2] W. Tybor, Acta Phys. Pol. B18, 369 (1987) .

[3] J. Rembieliński, W. Tybor, Acta Phys.Pol. B22, 439 (1991) .

[4] J. Rembieliński, W. Tybor, Acta Phys.Pol. B22, 447 (1991) .

[5] J.D. Bjorken, S.D. Drell, Relatywistyczna teoria kwantów,(Relativistic Quantum Mechanics,Relativistic Quantum Fields),PWN,Warszawa (1985) ,p.433. 